\title{
ORIGINAL
}

\section{ENFERMEDAD MENINGOCÓCICA E IMPACTO DE LA VACUNACIÓN SISTEMÁTICA CON LA VACUNA CONJUGADA ANTIMENINGOCÓCICA C EN UN ÁREA SANITARIA DE ANDALUCÍA}

Concepción Cruz Rojo (1), Carmen García Gil (1), Juan Nieto Vera (2) y Ana Monroy Morcillo (2)

(1) Departamento de Ciencias Socio-Sanitarias, Facultad de Medicina, Universidad de Sevilla.

(2) Distrito sanitario de Atención Primaria «Bahía de Cádiz-La Janda», Cádiz.

\section{RESUMEN}

Fundamento: En la Comunidad Autónoma de Andalucía, comenzó en julio de 2000 la vacunación con vacuna conjugada antimeningocócica $\mathrm{C}$ de todos los niños nacidos con posterioridad al 1 de enero de 1995, y en septiembre de 2001 se amplío a los nacidos a partir del 1 de enero de 1991, esto es, hasta los 10 años de edad. El objees valorar la efectividad de esta ntroducción masiva en los niños del área geográfica de un distrito sanitario, midiendo su impacto.

Métodos: Mediante un estudio longitudinal retrospectivo de incidencia poblacional se calculan las coberturas vacunales de los niños nacidos entre los años 1991 y 2001 y las tasas de incidencia de los casos declarados de enfermedad meningocócica en siete temporadas epidemiológicas (1997/98 a 2003/04). El impacto de la vacunación frente a la meningitis del serogrupo $\mathrm{C}$ se calcula comparando las tasas promedios anuales de las temporadas previas y posteriores a las campañas de vacunación en la población menor y mayor de 10 años, utilizando el test exacto de Fisher.

Resultados: En el periodo de estudio se declararon 109 casos de enfermedad meningocócica, de los que 50 eran de meningitis C. A partir de la temporada 2000-2001 descendió la incidencia de meningitis $\mathrm{C}$ en los niños menores de 10 años. En este grupo de edad se encuentran diferencias significativas entre las tasas promedio anual de las temporadas prevacunales y postvacunales (de 8,2 a 2,0 por 100.000 habitantes). En la población mayor de 10 años no se observa este descenso en la incidencia. En el periodo de estudio no se ha declarado ningún caso de fallo vacunal.

Conclusiones: La ausencia de fallos vacunales y la disminución de la incidencia de enfermedad meningocócica $\mathrm{C}$ en los niños menores de 10 años sugiere la buena efectividad de esta nueva vacuna conjugada frente al meningococo $\mathrm{C}$.

Palabras clave: Enfermedad meningocócica; Vacuna conjugada; Efectividad; Impacto vacunal.

Correspondencia:

Concepción Cruz-Rojo,

Departamento de Ciencias Socio-Sanitarias

Facultad de Medicina

Universidad de Sevilla

Avda. Sánchez Pizjúan s/n CP: 41009- Sevilla

Correo electrónico: cruzrojo@us.es
ABSTRACT

\section{Impact of Systematic Vaccination with the Antimeningococcal} C Conjugated Vaccine in a Health Area in Andalusia

Background: A retrospective longitudinal study of population incidence was made to assess the effectiveness of meningococcal serogroup $\mathrm{C}$ conjugate vaccine, after its mass introduction in children in the geographic area of a health district, measuring its popudren in the geographic area of a health district, measuring its popu-
lation impact, and we have studied the state of the meningococcal disease.

Methods: Vaccine coverage in children born between 1991 and 2001, and rates of incidence in declared cases of meningococcal disease in seven epidemiological seasons (1997/98 to 2003/04) were calculated. The impact of vaccination against serogroup $\mathrm{C}$ meningitis was assessed comparing the average annual rates of previous and later seasons to the vaccination campaigns in population younger and older than 10, using the Fisher exact test.

Results: In all the study period, 109 cases of meningococcal disease were declared, of which 50 were of serogroup $\mathrm{C}$ meningococcal disease. Starting from 2000/2001 season the incidence of serogroup $\mathrm{C}$ disease decreased in the population below 10 . In this age group, the annual average rate of post-vaccine seasons decreases in respect to pre-vaccine (from 8.2 to 2.0 per 100,000 inhabitants) showing a statistically significant difference. In the population above 10 years, this incidence reduction was not observed. In the study ve 10 years, this incidence reduction was not ob
period, no case of vaccine failure was declared.

Conclusions: The absence of vaccine failure and the impact observed on the incidence of serogroup $\mathrm{C}$ meningococcal disease in children under 10 suggests the effectiveness of this new conjugate vaccine, together with suitable vaccination conditions (vaccination schedule, high catch-up, etc.) which are developing in our health district.

Key words: Meningococcal disease. Conjugated vaccine. Effectiveness. Vaccine. 


\section{INTRODUCCIÓN}

La enfermedad meningocócica por Neisseria meningitidis es la más común de las meningitis bacterianas, endémica en nuestro medio en los meses de invierno con picos epidémicos debido a cambios antigénicos de la bacteria. En el conjunto de Andalucía, asî como en España, la meningitis predominante en los años ochenta era del serogrupo B. Tras un descenso de su incidencia condujo, a mediados de la decada de los 90, a un predominio del serogrupo $\mathrm{C}$ debido a un cambio de subtipo. Este nuevo patrón epidemiológico coincide con un aumento brusco de la incidencia de la meningitis $C^{1-4}$. Esta situación, que también ocurría en otros paises de nuestro entorno, llevó a intentar obtener una vacuna eficaz en los sectores de población más vulnerables a esta enfermedad: los niños y los adolescentes. En un primer momento se utilizó una vacuna antimeningocócica $\mathrm{A}+\mathrm{C}$ no conjugada, pero la escasa protección que ofrece esta vacuna en los niños más pequeños, unido a la corta duración de la inmunidad y a la falta de memoria inmunológica, hizo que se limitara su uso al control de brotes epidémicos y no se incluyera en el calendario de vacunaciones sistemáticas. En el área de estudio, esta vacuna únicamente se utilizó con ocasión de un brote que tuvo lugar en el año 1998 en el municipio de Puerto Real (Cádiz), vacunándose a los casi nueve mil niños y jóvenes de 2 a 18 años de edad.

Posteriormente se obtiene una vacuna conjugada que, aunque solo protege frente al meningococo del serogrupo $\mathrm{C}$, supera las limitaciones de la vacuna de polisacáridos ${ }^{5-7}$. En el año 1999 se inicia una campaña de vacunación masiva con esta vacuna dirigida a la población infantil y adolescente en Gran Bretaña ${ }^{8}$ y un año más tarde, en el año 2000, también se realizan campañas de vacunación y se introduce en el calendario de vacunación sistemática en España9 .

En el caso concreto de nuestra Comunidad Autónoma de Andalucía, en julio de
2000 comienza la vacunación a todos los niños nacidos con posterioridad al 1 de enero de 1995, y en septiembre de 2001 se amplía a los nacidos a partir del 1 de enero de 1991, esto es, hasta los 10 años de edad. En esta campaña masiva la pauta de vacunación cuando los niños tienen más de un año es de una dosis única. Además, la vacuna se introduce en el Calendario sistemático de vacunación infantil con una esquema de 3 dosis a los 2-4-6 meses.

El estudio de la eficacia y efectividad de la vacuna conjugada frente al meningococo del serogrupo $\mathrm{C}$, debería realizarse desde una visión global que abarque la valoración de la eficacia vacunal a través de estudios serológicos, la evaluación de los programas de vacunación y la medición del impacto poblacional sobre la incidencia de la enfermedad, que es la meta a la que se debe dirigir la introducción o administración sistemática de una vacuna ${ }^{10-14}$.

Además de estudiar la situación de la enfermedad meningocócica en los últimos años en el Distrito Sanitario «Bahía de Cádiz-La Janda» (área sanitaria de Andalucía), nuestro principal objetivo ha sido valorar, en un contexto epidemiológico, la efectividad de la vacuna conjugada antimeningocócica $\mathrm{C}$ tras su introducción masiva en los niños del área geográfica del Distrito, midiendo su impacto poblacional.

\section{MATERIAL Y MÉTODOS}

Se utiliza un diseño de seguimiento retrospectivo, analizando las diferencias en la incidencia de enfermedad meningocócica antes y después de una intervención preventiva como la vacunación.

El área geográfica del Distrito Sanitario «Bahía de Cádiz-La Janda» comprende 12 municipios con una población total de 492234 habitantes, según datos del Padrón Municipal de 2004 publicados por el Institu- 
to de Estadística de Andalucía ${ }^{15}$. Para calcular las coberturas vacunales se han utilizado datos procedentes del Departamento de Epidemiología del Distrito Sanitario. En el mismo se coordinan el Programa de Vacunación Infantil, a través de un «software» donde en sus bases de datos se encuentran las cohortes de niños que nacen en dicha área geográfica (obtenidas de los niños nacidos en todos los centros hospitalarios del área) y se especifican las dosis vacunales administradas durante los primeros años de su vida, y el Programa de Salud Escolar, que incluye la inmunización a escolares de 6 años de edad en adelante, y donde se evalúan las coberturas vacunales a partir de los listados de alumnos matriculados en todos los colegios de Enseñanza Primaria ubicados en el área de estudio, y que para esta vacuna comprendieron los cursos de $1^{\circ}$ a $6^{\circ}$ de Enseñanza Primaria (6 a 11 años). Al inicio del año 2002, tras las campañas masivas, se calcularon las coberturas de vacunación en las cohortes de niños nacidos entre los años 1991 y 2001.

A través del Sistema de Vigilancia Epidemiológica de Andalucía se disponen de todos los casos declarados de enfermedad meningocócica. La clasificación de los casos se realizó como sigue: a) casos de sospecha clínica, que se valoraron según los criterios de Boyer ${ }^{16}, b$ ) casos probables, que fueron definidos cuando un paciente presentaba síntomas clínicos compatibles con la enfermedad y un test analítico de presunción como la presencia intracelular de diplococos Gram negativo en el líquido cefalorraquídeo (LCR), y c) casos confirmados bacteriológicamente por cultivo del LCR o sangre, y serogrupados.

Se analizan siete temporadas epidemiológicas, las tres previas a la introducción de la vacuna conjugada antimeningocócica C (1997/98, 1998/99 y 1999/2000), y las cuatro temporadas siguientes comenzando en la 2000/01, donde se realizan las campañas de vacunación masiva a los niños menores de 10 años y en la que se introduce dicha vacuna en el Calendario de Vacunación infantil a los lactantes. Las temporadas epidemiológicas abarcan el período comprendido desde el 1 de Octubre de un año al 30 de Septiembre del año siguiente.

Se calculan las tasas de incidencia (por 100000 habitantes) en cada uno de los grupos de enfermedad meningocócica, utilizando como denominadores las poblaciones declaradas en el Padrón Municipal de habitantes correspondiente a cada uno de los años estudiados ${ }^{15}$, y el Censo de Población para el año $2001^{17}$. En cada temporada epidemiológica se ha utilizado la población del segundo año del periodo, que comprende nueve meses del año natural.

El impacto de la vacunación frente a la meningitis del serogrupo $\mathrm{C}$ se valora describiendo las tasas de incidencia, con sus intervalos de confianza al 95\%, de las siete temporadas epidemiológicas en la población objeto de la vacunación (niños menores de 10 años) y en la población mayor de 10 años.

Con el fin de aumentar la potencia estadística hemos agrupado las temporadas previas a la vacunación y las posteriores. Se ha utilizado la media aritmética de las poblaciones de los años 1998, 1999 y 2000 para calcular la tasa promedio anual en el periodo previo a la vacunación y la media aritmética de las poblaciones de los años 2001, 2002, 2003 y 2004 para la tasa promedio anual postvacunal. Para la comparación de ambas tasas promedio en los grupos de edad, se ha utilizado el test exacto de Fisher, considerándose las diferencias significativas para $\mathrm{p}<0.05$.

También hemos analizado la efectividad vacunal comprobando posibles fallos vacunales en las sospechas clínicas, los casos probables y en los casos confirmados de meningitis $\mathrm{C}$.

\section{RESULTADOS}

En la tabla 1 se presentan las coberturas vacunales frente a la meningitis $\mathrm{C}$ de los 
Tabla 1

Coberturas vacunales frente a meningitis $C$ en niños del área «Bahía de Cádiz-La Janda» tras las campañas de vacunación

\begin{tabular}{|c|c|cc|ccc|}
\hline \multirow{2}{*}{$\begin{array}{c}\text { Cohorte: } \\
\text { año de nacimiento }\end{array}$} & $\mathbf{N}^{\mathbf{0}}$ de niños & \multicolumn{3}{|c|}{$\begin{array}{c}\text { Niños bien } \\
\text { vacunados }\end{array}$} & \multicolumn{2}{|c|}{ Niños no vacunados } \\
& & $\mathbf{n}^{\mathbf{0}}$ & $\mathbf{\%}$ & $\mathbf{n}^{\mathbf{0}}$ & $\mathbf{\%}$ \\
\hline 2001 & 5173 & 5121 & 99,0 & $9 *$ & 0,2 \\
\hline 2000 & 4511 & 4444 & 98,5 & $14 *$ & 0,3 \\
\hline 1999 & 4798 & 4703 & 98,2 & 95 & 1,8 \\
\hline 1998 & 5256 & 5005 & 95,0 & 251 & 5,0 \\
\hline 1997 & 4946 & 4741 & 96,0 & 205 & 4,0 \\
\hline 1996 & 5024 & 4782 & 95,2 & 242 & 4,8 \\
\hline 1995 & 5508 & 5005 & 90,9 & 503 & 9,1 \\
\hline 1994 & 5577 & 5098 & 91,4 & 479 & 8,6 \\
\hline 1993 & 6112 & 5633 & 92,2 & 479 & 7,8 \\
\hline 1992 & 5837 & 5520 & 94,6 & 317 & 5,4 \\
\hline 1991 & 6828 & 6221 & 91,1 & 607 & 8,9 \\
\hline TOTAL & $\mathbf{5 9 5 7 0}$ & $\mathbf{5 6 2 7 3}$ & $\mathbf{9 4 . 5}$ & $\mathbf{3 2 0 1}$ & $\mathbf{5 . 4}$ \\
\hline
\end{tabular}

* En las cohorte del 2001 y del 2000 quedaron parcialmente vacunados $43(0.8 \%)$ y 53 niños (1.2\%), respectivamente, los cuales fueron excluidos del estudio.

Nota: Sólo los niños de estas dos cohortes, al ser menores de 1 año, necesitaron más de una dosis (esquema 2-4-6 meses).

niños objeto de las campañas de vacunación realizadas en los años 2000 y 2001. Destacan las altas coberturas, más del $98 \%$, obtenidas en los de niños más pequeños (nacidos en 1999, 2000 y 2001), observándose en el resto de cohortes cifras superiores al $90 \%$ de niños correctamente vacunados.

El total de casos de enfermedad meningocócica declarados en las temporadas analizadas ha sido 109, de los cuales 50 casos eran de meningitis del serogrupo C, 33 de meningitis B, 17 casos probables y 9 sospechas clínicas (ver tabla 2). La incidencia de enfermedad meningocócica ha experimentado un descenso desde la temporada epidemiológica 1999/2000, que presentó un máximo de 27 casos (tasa de 5.6 por 100000 habitantes), hasta la temporada 2003/2004 en la que se declararon 11 casos (tasa de 2.2). Esta tendencia viene definida por la meningitis $\mathrm{C}$, que aumenta su incidencia en la temporada 1999/2000 (16 casos, tasa de 3.3) para descender posteriormente, especialmente en las dos últimas temporadas (tasas de 0.6 y 3 casos cada una). Este descenso no se observa en la incidencia de meningitis B. Se destaca, también, que las sospechas clínicas de enfermedad meningocócica disminuyen en el tiempo, predominando los casos confirmados por laboratorio y los casos probables en los últimos años (tabla 2).

Observando en la tabla 3 la situación de la meningitis $\mathrm{C}$ por grupos de edad, sobresale el descenso de su incidencia, a partir de la 
ENFERMEDAD MENINGOCÓCICA E IMPACTO DE LA VACUNACIÓN SISTEMÁTICA CON LA VACUNA CONJUGADA...

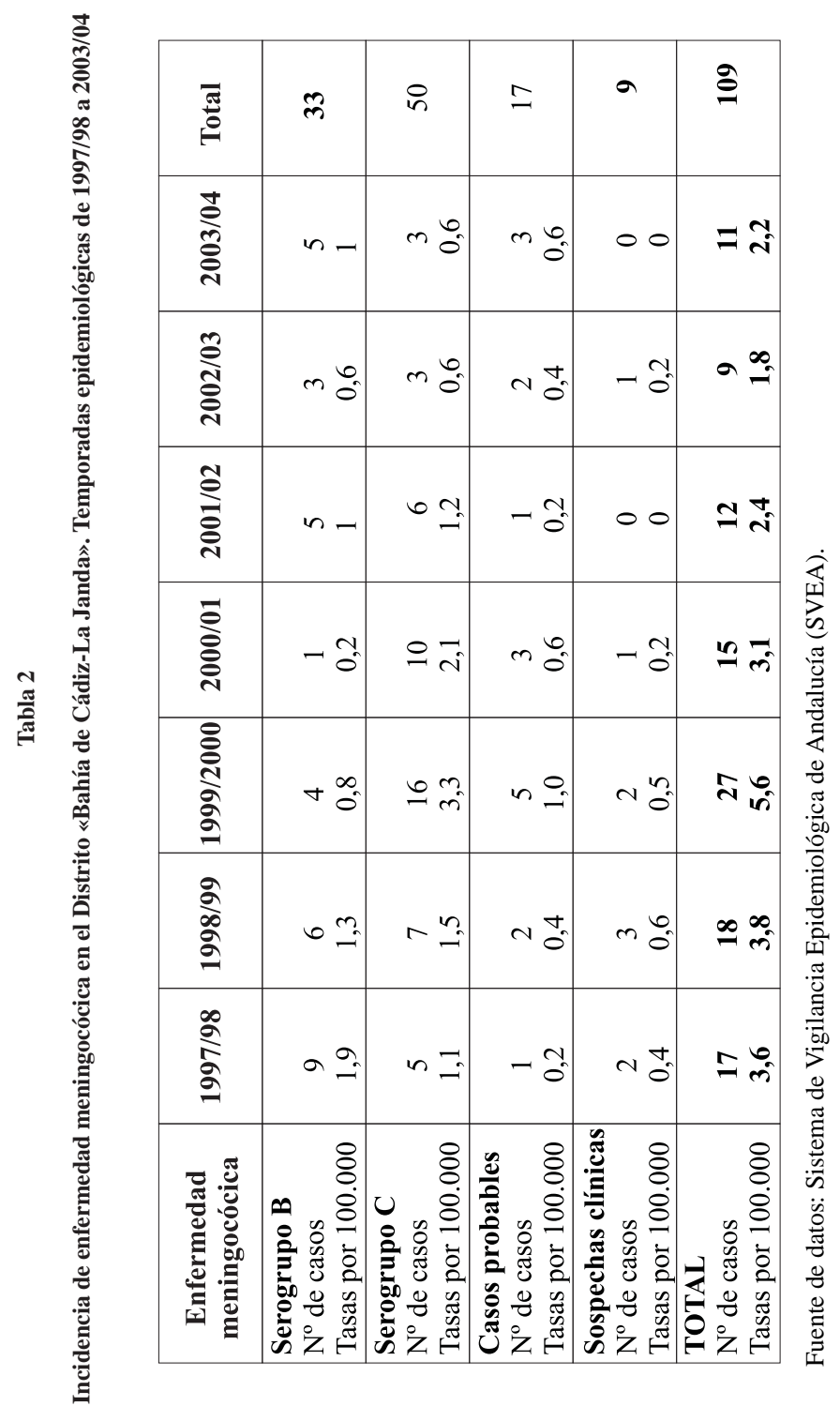




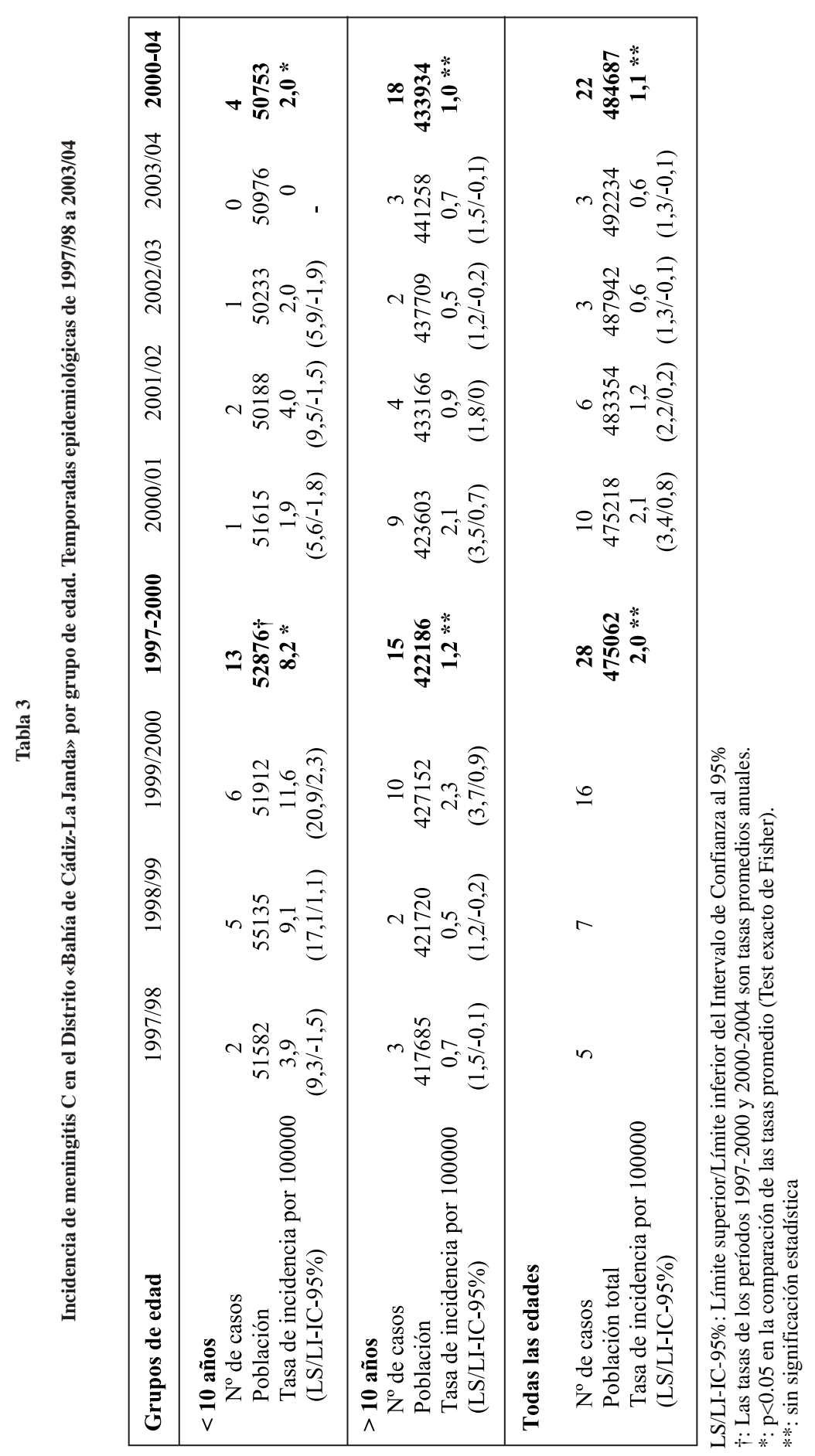


temporada 2000/2001, en la población objeto de la vacunación (menores de 10 años). Además, en este grupo de edad destaca el descenso de las tasas promedios anuales, declarándose en las temporadas prevacunales 13 casos de meningitis $C$ (tasa de 8.2 por 100.000), que pasaron a 4 casos en las temporadas postvacunales (tasa de 2.0 por 100.000 habitantes), siendo esta diferencia estadísticamente significativa. Por el contrario, en la población mayor de 10 años no se ha producido ni esa tendencia de descenso en la incidencia, ni una diferencia significativa entre las tasas promedios anuales de ambos períodos (tasas de 1.2 y 1.0 , respectivamente).

No se ha declarado ningún caso de meningitis C, ni de casos probables o sospechas clínicas de enfermedad meningocócica, que estuvieran previamente inmunizados con la vacuna conjugada.

\section{DISCUSIÓN}

La situación de la enfermedad meningocócica en la población del Distrito Sanitario «Bahía de Cádiz-La Janda» muestra un incremento claro de la meningitis $\mathrm{C}$ en la temporada epidemiológica 1999/2000, tras lo cual se produce un descenso de la incidencia de esta enfermedad hasta llegar a unas tasas muy bajas en las dos últimas temporadas, mientras que esta tendencia no se aprecia en la meningitis $\mathrm{B}$, como se ha descrito a nivel nacional ${ }^{18}$.

Las campañas de vacunación masivas y la introducción de la vacuna conjugada en el Calendario vacunal infantil, junto con las altas coberturas vacunales obtenidas (rango de $90.9 \%$ a $99 \%$ ), podrían explicar el descenso en la incidencia de la meningitis $\mathrm{C}$. Esta sospecha se acentúa cuando se analiza la tendencia en la población menor de 10 años, que fue objeto de la vacunación, donde se aprecia un descenso en el número de casos y en las tasas a partir de la temporada
2000/2001, y de forma más clara y significativa cuando se comparan las tasas promedio del periodo prevacunal con las del posvacunal. Reducciones similares se han encontrado en otros estudios de impacto realizados en Andalucía, España y otros países, y que se atribuyen también a la introducción masiva de la nueva vacuna conjugada frente a la meningitis $\mathrm{C}^{18-23}$. Sin embargo, no debemos olvidar las ondas típicas de la enfermedad, ya que el aumento en el número de casos en la temporada 1999/2000 podría coincidir con un pico de la onda y los años posteriores con la bajada correspondiente. Sería necesario analizar la evolución de esta enfermedad con una perspectiva de más años para confirmar que la vacunación ha roto, como ha ocurrido con otras enfermedades vacunables, las ondas típicas y naturales de esta enfermedad infecciosa ${ }^{5}$. Una observación que apunta en dirección contraria a que este descenso de la incidencia de la meningitis $\mathrm{C}$ sea debida a una evolución natural de la enfermedad, lo proporciona el que esta tendencia no se presente en la población de mayores de 10 años.

Otra posible limitación a los resultados de este estudio lo constituye el hecho de estudiar un área geográfica relativamente pequeña y una enfermedad con una baja incidencia anual, lo que ha restado precisión en nuestros análisis estadísticos, y especialmente en el grupo de población menor de 10 años. Esta limitación se había previsto y para minimizarla se procedió a agrupar las temporadas para incrementar la potencia estadística.

Por otro lado, este descenso de la incidencia de la meningitis $\mathrm{C}$ se ve reforzado por la mejora del diagnóstico microbiológico que provoca una disminución de la incidencia de las sospechas clínicas de enfermedad meningocócica de las últimas temporadas epidemiológicas y un incremento porcentual de las meningitis serogrupadas sobre el total de enfermedad meningocócica declarada, lo que hace que sea más real la incidencia de las meningitis C y B de los últimos años. 
A pesar de que en otros estudios se han señalado algunos casos, aunque poco significativos, de fallos vacunales ${ }^{18,23}$, en los resultados de este estudio se muestra la ausencia total de fallos vacunales en todos los casos declarados de meningitis $\mathrm{C}$ y en las sospechas clínicas y casos probables de enfermedad meningocócica. Esto se puede deber al tamaño relativamente pequeño de nuestra área geográfica, pero también puede mostrar una mejora de la efectividad de la vacuna cuando se administra en las mejores condiciones sanitarias (control de la cadena de frío, número de dosis adecuado según la edad y el seguimiento estricto con el fin de que los lactantes reciban las tres dosis necesarias para su correcta inmunización: 2-4-6 meses). En una reciente publicación en Inglaterra sobre la efectividad vacunal a largo plazo $^{22}$ se ha observado un mayor número de fallos vacunales en los niños que recibieron la pauta vacunal de 3 dosis a los 2-34 meses de edad, lo que ha originado el cuestionamiento de dicho esquema, planteándose el establecimiento de una cuarta dosis o la administración de la tercera en una edad más tardía.

Los resultados de este estudio sugieren que la vacunación en el área del Distrito estudiado está teniendo un impacto efectivo en los niños menores de 10 años. Sin embargo, no hemos podido constatar este mismo efecto en el resto de la población, aunque se conoce que una medida preventiva como la inmunización tiene efectos beneficiosos de forma indirecta a los no vacunados, a través del llamado «efecto rebaño». Así, en un estudio reciente realizado en Inglaterra se concluye que la vacuna conjugada antimeningocócica $\mathrm{C}$, tras la vacunación masiva a su población de niños y jóvenes, contribuye al control de la enfermedad meningocócica mediante una protección indirecta, reduciendo un $67 \%$ la tasa de ataque en la población no vacunada y proporcionando evidencias de la inmunidad de rebaño sobre la población en su conjunto ${ }^{24}$.
Los hallazgos obtenidos en el presente estudio nos hacen ser optimistas sobre la efectividad de esta nueva vacuna conjugada frente al meningococo $\mathrm{C}$, y nos animan a continuar su administración con las mejores garantías de calidad y captando al máximo de población objeto de esta vacuna, que en Andalucía está siendo incrementada periódicamente para cubrir a toda la población infantil y adolescente de nuestra Comunidad Autónoma.

\section{BIBLIOGRAFÍA}

1. Centro Nacional de Epidemiología. Enfermedad meningocócica. Situación en la temporada 19981999. Bol Epidemiol Sem 1999;7(21):221-232.

2. Mateo S, Cano R, García C. Changing epidemiology of meningococcal disease in Spain, 19891997. Euro Surveill 1997;2:71-74.

3. Larrauri A, Cano R, García M, Mateo S. Enfermedad meningocócica en España. Temporada 20022003. Impacto de la vacuna conjugada en las tres temporadas posterior a su introducción en España. Bol Epidemiol Sem 2004;2(12):13-24.

4. Boletín Epidemiológico de Andalucía. Informe Semanal. Enfermedad Meningocócica. Año 2002. Bol Epidemiol Sem 2003;33(8).

5. Comité Asesor de Vacunas. Manual de Vacunas en Pediatría. Madrid: Asociación Española de Pediatría; 2001.

6. Choo S, Zuckerman J, Goilav C, Hatzmann E, Everard J, Finn A. Immunogenicity and reactogenicity of a group $\mathrm{C}$ meningococcal conjugate vaccine compared with a group $\mathrm{A}+\mathrm{C}$ meningococcal polysaccharide vaccine in adolescents in a randomised observer-blind controlled trial. Vaccine 2000;24(18):2686-2692.

7. Jódar L, Griffiths E, Feavers I. Scientific challenges for the quality control and production of group $\mathrm{C}$ meningococcal conjugate vaccines. Vaccine 2004;22(8);1047-1053.

8. Ramsay ME, Andrews N, Kaczmarski EB, Miller E. Efficacy of meningococcal sero-group $\mathrm{C}$ conju-

Rev Esp Salud Pública 2005, Vol. 79, N. ${ }^{\circ} 6$ 
gate vaccine in teenagers and toddlers in England. Lancet 2001;357: 195-6.

9. Dirección General de Farmacia y Productos Sanitarios. Ministerio de Sanidad y Consumo. Circular $12 / 2000$.

10. Matter HC, Cloetta J, Zimmermann H, Sentinella A. Measles, mumps, and rubella: monitoring in Switzerland through a sentinel network, 19861994. J Epidemiol Community Health 1995;49 (suppl 1):4-8.

11. Flores R, Cremades A. Brote de parotiditis en el área sanitaria 17. Eficacia de la vacuna triple vírica (II) y comparación de la incidencia de la enfermedad según el año de vacunación. Rev Esp Salud Pública 1998;72:123-4.

12. Peñuelas JA, Peyró R, Diestro A, Pastor MC. Efectividad vacunal, según las cepas administradas en un brote epidémico de parotiditis. Rev Esp Salud Pública $1998 ; 72: 130$.

13. Chamot E, Toscani L, Egger P, Germann D, Borquin C. Estimation de l'efficacité de tríos souches vaccinales ourliennes au cours d'une épidémie d'oreillons dans le canton de Genève. Rev Epidemiol Santé Publique 1998;46:100-107.

14. Rojo CC, Iglesias MR, Olvera CJ, Alvarez M. Study of the immune response engendered by differents combined measles, mumps and rubella (MMR) vaccines in an area of Andalusia (Spain). Vaccine 2003;22:280-286.

15. http://www.juntadeandalucia.es/institutodeestadistica/

16. Thomé J, Bovier-Lapierre M, Verherat M. Méningite bactérienne ou méningite virale? Etude d'un sore permettant une orientation étiologique precoce dans les meningites de diagnostic difficile. Pédiatrie 1980;35:225-36

17. Instituto Nacional de Estadística. Censo de la Población Española 2001. Madrid: INE; 2003.

18. Cano R, Larrauri A, Mateo S, Alcalá B, Salcedo C, Vázquez JA. Impact of the meningococcal C conjugate vaccine in Spain: an epidemiological and microbiological decision. Euro Surveill 2004;9(7):5-6

19. Balmer P, Borrow R, Miller E. Impact of meningococcal $\mathrm{C}$ conjugate vaccine in UK. J Med Microbiol 2002;51:717-722.

20. Trotter CL, Ramsay ME, Kaczmarski EB. Meningococcal serogroup $\mathrm{C}$ conjugate vaccination in England and Wales: Coverage and initial impact of the campaign. Common Dis Public Health 2002;5:220-5.

21. Mooney JD, Christie P, Robertson C, Clarke SC. The impact of meningococcal serogroup $C$ vaccine in Scotland. Clin Infect Dis 2004; 39 (3):349-56.

22. Trotter CL, Andrews NJ, Kaczmarski EB, Miller E, Ramsay ME. Effectiveness of meningococcal serogroup $\mathrm{C}$ conjugate vaccine 4 years after introduction. Lancet 2004; 364(9431):365-7.

23. De Walls PD, Deceunick G, Boulianne N, De Serres G. Effectiveness of a mass immunization campaign using serogroup $\mathrm{C}$ meningococcal conjugate vaccine. JAMA 2004; 292(20):2491-4.

24. Ramsay ME, Andrews NJ, Trotter CL, Kaczmarski EB, Miller E. Herd immunity from meningococcal serogroup $\mathrm{C}$ conjugate vaccination in England: database análisis. BMJ 2003;326:365-366. 